\title{
Connective Tissue Disease-Associated Interstitial Lung Disease
}

\section{A Call for Clarification}

\author{
Aryeh Fischer, MD; Sterling G. West, MD; Jeffrey J. Swigris, DO; \\ Kevin K. Brown, MD; and Roland M. du Bois, MD
}

\begin{abstract}
This commentary highlights the present dilemmas surrounding the classification of a patient with interstitial pneumonia who has clinical features suggesting an associated connective tissue disease but the features fall short of a clear diagnosis of connective tissue disease-associated interstitial lung disease under the current rheumatologic classification systems. This commentary illustrates what we perceive to be the limitations in the present approach to the classification of this group of patients and discusses problems with redefining the diagnosis of undifferentiated connective tissue disease to encompass patients with interstitial pneumonia. Finally, we advocate not only for a multidisciplinary approach to evaluation, but also disease classification and offer a proposal to define them as a distinct phenotype-lung-dominant CTD—for which prognostic, therapeutic, and pathobiologic implications can be tested in future, hopefully multiinstitutional, studies.

CHEST 2010; 138(2):251-256
\end{abstract}

\begin{abstract}
Abbreviations: $\mathrm{ANA}=$ antinuclear antibody; $\mathrm{CCP}=$ cyclic citrullinated peptide; $\mathrm{CTD}=$ connective tissue disease; CTD-IP = connective tissue disease-associated interstitial pneumonia; IIP = idiopathic interstitial pneumonia; ILD = interstitial lung disease; IP = interstitial pneumonia; LIP = lymphocytic interstitial pneumonia; NSIP = nonspecific interstitial pneumonia; $\mathrm{RF}=$ rheumatoid factor; $\mathrm{RNP}=$ ribonucleoprotein; $\mathrm{SSc}=$ systemic sclerosis; $\mathrm{UCTD}=$ undifferentiated connective tissue disease; UIP = usual interstitial pneumonia
\end{abstract}

$\mathbf{W}$ hether the idiopathic interstitial pneumonias (IIPs) are actually a pulmonary manifestation of an underlying systemic autoimmune disease (ie, connective tissue disease [CTD]) is a recurring question for those involved in the clinical care or research of patients with interstitial lung disease (ILD). Most tellingly are a large number of patients who are unclassifiable and yet seem to fit at the interface of the two disorders, that is, those in whom it appears that the lung is either the lone or the most dominant clinically significant manifestation of an occult CTD. We believe that our current strategies for identifying and classifying these patients are inadequate.

Manuscript received January 22, 2010; revision accepted March 12, 2010.

Affiliations: From the Interstitial and Autoimmune Lung Disease Program (Drs Fischer, Swigris, Brown, and du Bois), National Jewish Health, and Division of Rheumatology (Drs Fischer and West), National Jewish Health and University of Colorado, Denver, CO.

Correspondence to: Aryeh Fischer, MD, National Jewish Health, 1400 Jackson St, Denver, CO 80206; e-mail: fischera@ njhealth.org
It is not uncommon to find certain patients with interstitial pneumonia (IP) who are suspected to have a systemic autoimmune disease based on the presence of circulating autoantibodies, specific histopathologic features, or subtle extrathoracic manifestations. However, within current classification schemes, many of these patients are labeled as idiopathic by default because they do not meet accepted rheumatologic criteria for a defined CTD. In addition, despite the recognition that IP may be the forme fruste presentation of systemic autoimmune disease, current classification criteria do not allow a CTD designation for ILD in isolation.

This commentary highlights the present dilemmas surrounding the classification of a patient with IP who has clinical features that suggest an associated CTD, but the features fall short of allowing a clear diagnosis

() 2010 American College of Chest Physicians. Reproduction of this article is prohibited without written permission from the American College of Chest Physicians (http://www.chestpubs.org/ site/misc/reprints.xhtml).

DOI: $10.1378 /$ chest.10-0194 
of CTD-associated IP (CTD-IP) to be made under the current classification systems. We illustrate what we perceive to be the limitations in our present approach 49pose to define these conditions as a distinct phenotype-"lung-dominant CTD"-for which prognostic, therapeutic, and pathobiologic implications can be tested in future, hopefully multiinstitutional, studies.

We offer these concepts as an initial step of an interactive process with an aim to more effectively engage multidisciplinary and multiinstitutional collaboration and create a framework for further investigation of a poorly understood spectrum of IP that has a rheumatologic flavor and yet cannot presently be defined as CTD-IP. We acknowledge that few data would allow an immediate change in classification, but there are sufficient leads to support the need for this to be explored more comprehensively. As with other classification systems such as that for the IIPs, we anticipate that this characterization will evolve iteratively over time as a consequence of the outcome of studies that we hope to encourage.

\section{IMPLICATIONS OF THE DIAGNOSIS}

In our opinion, numerous implications of identifying underlying systemic autoimmune disease in patients presenting with an IP exist. Most significantly, CTD-IP is associated with a more favorable prognosis than IIP. ${ }^{1}$ Although it is not known whether the identification of occult forms of CTD carries a similarly more-favorable prognosis, it can be argued that future decisions that incorporate the knowledge of underlying autoimmunity might well promote an improved understanding of pathogenesis and better treatment choices. We hypothesize that identifying patients with occult or incomplete forms of CTD-IP and classifying these forms as a unique phenotype of "lungdominant CTD" will enable more precise disease classification; provide a platform by which collaborative investigations can be performed; and provide valuable answers to questions of pathobiology, natural history, and therapeutic responsiveness.

\section{Challenges AND Limitations}

Although all pulmonologists see patients in whom they suspect an underlying autoimmune mechanism as the cause of the pulmonary disease, confirming systemic autoimmune disease and diagnosing specific CTDs in the absence of classic clinical findings are challenging,,$^{2,3}$ and we believe that current screening strategies are largely inadequate. Detecting CTD by simply screening with nonspecific autoantibodies does not suffice, ${ }^{2-6}$ and current rheumatologic classification schemes are limiting because they do not allow a CTD designation to be applied when IP is the lone manifestation. Indeed, classification schemes for the CTDs are based on specific extrathoracic symptoms and signs and the presence of specific serologic autoantibodies. Although IP is a well-known manifestation of CTD and occurs commonly in this context, it is not included in the diagnostic criteria for any of the CTDs except as a minor criterion for systemic sclerosis (SSc). Further, to our knowledge, isolated IP (ie, without extrathoracic signs or symptoms of CTD) never has been formally considered as a possible lone manifestation of CTD, and as such, a great number of patients with isolated IP are classified as idiopathic by default. We find it problematic that, for example, a 30-year-old woman with keratoconjuctivitis sicca and a positive anti-Ro antibody is defined as having CTD (Sjögren syndrome), but a 30-year-old woman with a positive anti-Ro antibody and biopsyproven nonspecific interstitial pneumonia (NSIP) with overlapping histopathologic features of organizing pneumonia, lymphoid follicles with germinal centers, extensive pleuritis, and dense perivascular collagen is labeled with idiopathic rather than CTD-IP.

These cases highlight the following dilemma: Classification schemes for the CTDs require the presence of certain extrathoracic features combined with the presence of specific autoantibodies. As such, unless extrathoracic features are identified that allow a precise CTD designation to be made, patients with IP and lung features suspicious for CTD remain unclassifiable.

Far too often, pulmonologists and rheumatologists find themselves at odds over whether CTD is present to account for IP. Given the constraints of our limited knowledge of IP, both parties are likely correct. We believe that pulmonologists are correct in that the lung features (ie, the predominant histologic pattern and secondary features) themselves do not fit with idiopathic disease and that rheumatologists are correct in that patients with IP alone are not classifiable without extrathoracic manifestations.

\section{IDENTIFYING OCCULT CTD}

We believe that the detection of occult CTD in patients presenting with IP is optimized by multidisciplinary collaboration. Finding occult CTD is not uncommon. It has been estimated that among patients presenting with an apparently IIP, roughly $15 \%$ are found to have underlying CTD after more thorough evaluation. ${ }^{5}$ Homma and colleagues ${ }^{7}$ evaluated whether IP as the sole presentation of CTD can be differentiated from IIP. They described 68 patients who had presented with IIP and were followed prospectively for $>11$ years. Thirteen (19\%) patients eventually developed classifiable CTD. The prevalence of a positive rheumatoid factor $(\mathrm{RF})$ or antinuclear antibody 
(ANA) was no different in the group that developed CTD than in the group that did not. The authors concluded that patients who are defined as having an IIP cannot be distinguished from those with CTD-IP before the systematic manifestations appear. Although we support their findings that detecting occult CTD is challenging and that $\mathrm{ANA}$ and $\mathrm{RF}$ positivity alone are not very useful in this endeavor, we offer an alternative set of conclusions: A thorough evaluation for subtle extrathoracic features of underlying CTD, assessment for more specific autoantibodies (and incorporating ANA titer and pattern of immunofluorescence), and consideration of histopathologic features are important components of an IP evaluation and make it more likely that occult CTD will be detected.

\section{Autoantibodies}

Despite our knowledge that detecting CTD in patients presenting with IP is important and common, there are no protocols or guidelines to aid the clinician. The current practice is to draw an ANA and $\mathrm{RF}$ (and occasionally other autoantibodies) in patients presenting with an IP, and if any are positive, a rheumatologic evaluation is requested. In our opinion, this approach is less than ideal for a number of reasons. Foremost, ANA and RF are poor screening tests because they have low specificity, particularly when present at low titer, and can be seen in healthy individuals. In addition, given that a negative ANA and $\mathrm{RF}$ may dissuade some clinicians from pursuing further evaluation, cases of occult CTD that may be ANA and RF negative (eg, antisynthetase syndrome) are missed. We recently described a cohort of patients with antisynthetase syndrome who presented with IP and were characteristically ANA negative. ${ }^{8}$ All patients had subtle features of the antisynthetase syndrome (eg, mechanic's hands), prompting further testing for rare but confirmatory anti-tRNA synthetase antibodies. Prior to evaluation at our center, none of the patients had undergone further evaluation for CTD, and we suspect that this was due to the negative ANA, RF, and ANA profile that had been performed elsewhere as screening tests.

More-specific antibodies serve as integral components in the assessment for CTD-IP, and often, practitioners need to order more than an ANA and RF to screen for CTD more effectively. Furthermore, we believe that it is important to take note of the pattern of immunofluorescence when the ANA is positive because the nucleolar-staining ANA pattern in patients with IP suggests SSc spectrum of disease. ${ }^{9}$ Particularly helpful antibodies to request in patients with IP as part of a CTD evaluation include anti-Scl-70, anti-tRNA synthetase antibodies (eg, Jo-1, PL-7, PL-12), anti-Ro (SS-A), antiribonucleoprotein (anti-
RNP) and anti-cyclic citrullinated peptide (anti-CCP). Anti-Scl-70 is highly specific for SSc, and anti-Ro is present in a broad array of CTDs characteristically associated with IP (eg, Sjögren syndrome). High-titer anti-RNP supports a diagnosis of mixed CTD, antitRNA synthetase antibodies confirm the antisynthetase syndrome, and anti-CCP antibodies (in contrast to the nonspecific RF) are highly specific for rheumatoid arthritis.

\section{Is It Really CTD? It Depends on Whom You Ask}

Paradoxically, the detection of autoantibodies often raises more questions and may not clarify classification. In the absence of extrathoracic manifestations attributable to CTD (eg, inflammatory arthritis, sclerodactyly), rheumatologists are reluctant to diagnose CTD, even when highly specific autoantibodies and a fitting IP pattern are present. To illustrate this point, consider a 35-year-old man with a positive anti-RNP antibody, fibrotic NSIP, and no extrathoracic features of systemic autoimmunity. Under the existing scheme, this patient would not be classified as having CTD. Similarly, a 40-year-old woman with lymphoid interstitial pneumonia (LIP), a high-titer speckled ANA, and esophageal hypomotility as her only extrathoracic abnormality would not be defined as having CTD. Neither case meets diagnostic criteria for CTD or would be considered as idiopathic by pulmonologists. This situation is frequent but not acceptable because everyone, including most importantly the patient, is left with an often distressing uncertainty. On the basis of current classification schemes, such cases are presently classified (we would actually say misclassified) as idiopathic or, worse, left unclassified.

\section{Histopathologic Considerations}

Careful review of the histopathology from surgical lung biopsy specimens may provide clues that the IP is a manifestation of underlying CTD. There are a number of suggestive patterns and features observed on histopathology with known associations with CTD. ${ }^{10-13}$ A higher index of suspicion for occult CTD is warranted in any case of NSIP or LIP because these are two of the more commonly recognized patterns in patients with CTD. In addition, secondary histopathologic features that should raise strong suspicions for underlying CTD include dense perivascular collagen, extensive pleuritis, lymphoid aggregates with germinal center formation, and prominent plasmacytic infiltration. ${ }^{10-12,14}$ These histologic features alert the pathologist that the injury pattern is more likely due to an underlying CTD. In such cases, the pulmonologist may conclude that the biopsy specimen is consistent with CTD-IP and choose to manage the 
patient as such. In contrast, rheumatologists are less apt to label a patient as having CTD when extrathoracic manifestations are absent no matter what the lung histology shows.

Recent data suggest that the presence of circulating autoantibodies is associated with specific histopathologic findings, even in the absence of characterizable CTD. Song and colleagues compared and contrasted secondary histopathologic features among three groups of patients with usual interstitial pneumonia (UIP) pattern lung injury. Group $1(\mathrm{n}=39)$ comprised subjects with CTD-UIP; group $2(\mathrm{n}=27)$, subjects who had idiopathic UIP with ANA or RF positivity; and group $3(\mathrm{n}=34)$, subjects who had idiopathic UIP and were antibody negative. Presumably, the subjects in group 2 (antibody-positive, idiopathic UIP) were not considered as having CTD based on the absence of characterizable extrathoracic features or more specific autoantibodies. Among those with CTD-UIP were more germinal centers, plasma cells, and fewer fibroblastic foci than that found in all subjects with idiopathic UIP. Interestingly, however, histopathologic features differed between the subgroups of idiopathic UIP (groups 2 and 3) based on autoantibody status. Although none of the subjects with antibody-positive idiopathic UIP (group 2) had extrathoracic features of CTD, they had higher germinal center scores and more plasma cells than subjects with antibody-negative idiopathic UIP (group 3). Notably, no histopathologic features distinguished CTD-UIP (group 1) from antibody-positive idiopathic UIP (group 2). Among those subjects with idiopathic UIP (groups 2 and 3), antibody status did not affect survival, although as the authors point out, this might have been due to the small sample sizes in each group, but those with idiopathic UIP had a worse prognosis than those with CTD-UIP (group 1). The impact of circulating, albeit nonspecific, autoantibodies on the underlying histopathologic features is of interest and raises the possibility that systemic autoimmunity may be of etiologic importance in this cohort. The significance of these findings is not known, but we believe that they merit further investigation.

\section{REDEFINING UNDIFFERENTIATED CTD Is PRoblematic}

The concept has been proposed that all patients with idiopathic NSIP, even those without extrathoracic features or serum autoantibodies, actually have an undifferentiated CTD (UCTD). ${ }^{13,15}$ In our opinion, although this hypothesis is interesting, the revised application of this CTD diagnosis to encompass all NSIP cases is problematic. Redefining the UCTD diagnosis in this way requires input from rheumatologists who are generally skeptical about accepting IP as a diagnostic criterion for CTD. For example, rheumatologists would classify a 55-year-old woman with inflammatory arthritis and a positive ANA as UCTD but not a 55-year-old woman with NSIP and a positive ANA. Rheumatologists do not consider patients with isolated NSIP to have CTD unless there are extrathoracic features present (eg, Raynaud phenomenon, inflammatory arthritis) and specific autoantibodies to lend more certainty to the CTD label. Another difficulty with use of the term "UCTD" is that rheumatologists consider UCTD to be indicative of mild disease. ${ }^{16,17}$ To attempt to redefine UCTD to include isolated, and often life-threatening, IP is unlikely to succeed because we strongly suspect it would not be adopted by rheumatologists. In essence, the term "UCTD" is well established within the rheumatology community, reflects evolving or partial presentation of milder forms of CTD, and is not manifested by ILD or other organ-threatening disease.

\section{Some Proposed Solutions}

In the face of the challenges posed by these disorders that fall short of universally acceptable diagnoses and of discordant perceptions about disease classification as well as the impasse in accepting the redefining of UCTD, we offer the following modest suggestions.

\section{Multidisciplinary Collaboration}

Just as the evaluation of patients with IP is optimized by a multidisciplinary approach, we advocate for a multidisciplinary approach to the classification of occult forms of CTD. The designation of CTD-IP requires consensus involving both the pulmonary and the rheumatology communities. Presently, far too little interdisciplinary dialogue occurs in this arena, and the advancement of this field would be better served by efforts to bridge this divide. Having a consensus from both the pulmonary and the rheumatology communities on precise CTD disease characterization and classification should provide a significant step in the right direction toward addressing the interdisciplinary roadblock.

\section{Consider Lung-Dominant CTD}

A novel classification scheme may provide an avenue of consensus between pulmonologists and rheumatologists and will allow our hypotheses to be tested. With this aim in mind, we suggest use of the term "lung-dominant CTD" CTD for cases where IP has a rheumatologic flavor as supported by specific autoantibodies or histopathologic features and yet does not meet criteria for a defined CTD based on the lack of adequate extrathoracic features to confer a diagnosis of definite CTD. Implicit with the proposed term 
Table 1-Proposed Provisional Criteria for Lung-Dominant CTD

1. NSIP, UIP, LIP, OP, and DAD (or DIP if no smoking history), as determined by surgical lung biopsy specimen or suggested by high-resolution CT and

2. Insufficient extrathoracic features of a definite CTD to allow a specific CTD designation and

3. No identifiable alternative etiology for IP and

4. Any one of the following autoantibodies or at least two of the histopathology features:

$\begin{array}{ll}\begin{array}{l}\text { Autoantibodies } \\ \text { a. High-titer ANA }(>1: 320) \text { or }(>60 \mathrm{IU} / \mathrm{mL})\end{array} & \begin{array}{c}\text { Histopathology features } \\ \text { (a) Lymphoid aggregates with } \\ \text { germinal centers }\end{array} \\ \begin{array}{l}\text { b. Nucleolar-ANA } \\ \text { c. Anti-CCP }\end{array} & \begin{array}{c}\text { (b) Extensive pleuritis } \\ \text { (c) Prominent plasmacytic } \\ \text { infiltration }\end{array} \\ \begin{array}{l}\text { d. Anti-Scl-70 } \\ \text { e. Anti-Ro }\end{array} \\ \begin{array}{l}\text { f. Anti-La } \\ \text { g. Anti-dsDNA }\end{array} \\ \begin{array}{l}\text { h. Anti-Smith } \\ \text { i. Anti-RNP } \\ \text { j. Anti-tRNA synthetase (eg, perivascular collagen } \\ \quad \text { Jo-1, PL-7, PL-12, and } \\ \quad \text { others) }\end{array} \\ \text { k. Anti-PM-Scl } \\ \text { l. Anticentromere }\end{array}$

$\mathrm{ANA}=$ antinuclear antibody; $\mathrm{CCP}=$ cyclic citrullinated peptide; $\mathrm{CTD}=$ connective tissue disease $\mathrm{DAD}=$ diffuse alveolar damage; $\mathrm{DIP}=$ desquamative interstitial pneumonia; $\mathrm{IP}=$ interstitial pneumonia; LIP $=$ lymphocytic interstitial pneumonia; NSIP $=$ nonspecific interstitial pneumonia; $\mathrm{OP}=$ organizing pneumonia; $\mathrm{RF}=$ rheumatoid factor; $\mathrm{RNP}=$ ribonucleoprotein; $\mathrm{UIP}=$ usual interstitial pneumonia.

"lung-dominant CTD" is the recognition that specific autoantibodies and histopathologic features alone can be enough to characterize and classify a patient as having a CTD-IP. The presence of objective extrathoracic features highly suggestive of CTD (eg, Raynaud phenomenon, esophageal hypomotility, inflammatory arthritis of the metacarpophalangeal joints or wrists, digital edema, symptomatic keratoconjuctivitis sicca ) are important and will lend further support for an underlying CTD, but their absence should not preclude a diagnosis of lung-dominant CTD. Although these extrathoracic features lend support for a diagnosis of CTD, they can be affected by subjective interpretation, and therefore, their presence in a patient with IP in the absence of histopathologic or serologic evidence of CTD is not sufficient to confer a designation of lung-dominant CTD.

In Table 1, we propose a set of diagnostic criteria for what we would consider to be lung-dominant CTD. We hope that this concept and these criteria are viewed as provisional because no evidence base supports the immediate use of such a classification, and it might serve as a platform for further multidisciplinary investigation, including validation through prospective study.

There are a number of advantages to the introduction of this novel classification. First, the criteria offered are objective and measurable. Second, nonspecific symptoms (eg, myalgias, arthralgias, esophageal reflux disease), nonspecific inflammatory markers (eg, erythrocyte sedimentation rate), and low-titer ANA or RF are not included because all occur commonly in patients without definite CTD. Third, the term "lung-dominant CTD" conveys that this entity is distinct from the idiopathic group of IP and acknowledges that the IP has the "feel" of CTD as manifested by systemic autoimmunity and yet cannot be designated more specifically as a definable CTD according to current classification systems used by rheumatology societies. Fourth, these patients require surveillance for evolution to more-defined CTD because recognition that lung-dominant CTD may evolve to definable CTD is implicit in our definition. Finally, conferring a diagnosis of lung-dominant CTD for these types of patients will remove them from the (default) category of IIP yet allow their distinction from more definite forms of CTD and provide a framework by which questions regarding this subset's natural history, pathobiology, treatment, and prognosis can be answered. We emphasize that the proposed classification of lung-dominant CTD be viewed as provisional and requiring validation with future research studies.

\section{CONCLUSIONS}

We believe that multidisciplinary collaboration in the evaluation of IP and testing the concept of lungdominant CTD ultimately will allow more precise disease differentiation to be made and might lead to a better understanding of IP. In addition, it can be argued that future decisions that incorporate the knowledge of lung-dominant CTD might well lead to the development of more targeted therapies as well as affect treatment choices. Further research is needed to validate the proposed provisional criteria, to determine the natural history of lung-dominant CTD, and to assess whether it is associated with a prognosis that is similar to more established forms of CTD-IP.

\section{ACKNOWLEDGMENTS}

Financial/nonfinancial disclosures: The authors have reported to CHEST that no potential conflicts of interest exist with any companies/organizations whose products or services may be discussed in this article.

\section{REFERENCES}

1. Park JH, Kim DS, Park IN, et al. Prognosis of fibrotic interstitial pneumonia: idiopathic versus collagen vascular disease-related subtypes. Am J Respir Crit Care Med. 2007;175(7):705-711.

2. Cottin V. Interstitial lung disease: are we missing formes frustes of connective tissue disease? Eur Respir J. 2006;28(5):893-896.

3. Tzelepis GE, Toya SP, Moutsopoulos HM. Occult connective tissue diseases mimicking idiopathic interstitial pneumonias. Eur Respir J. 2008;31(1):11-20. 
4. Antoniou KM, Margaritopoulos G, Economidou F, Siafakas NM. Pivotal clinical dilemmas in collagen vascular diseases associated with interstitial lung involvement. Eur Respir J. 2009;33(4):882-896.

5. Strange C, Highland KB. Interstitial lung disease in the patient who has connective tissue disease. Clin Chest Med. 2004;25(3):549-559.

6. Mittoo S, Gelber AC, Christopher-Stine L, Horton MR, Lechtzin N, Danoff SK. Ascertainment of collagen vascular disease in patients presenting with interstitial lung disease. Respir Med. 2009;103(8):1152-1158.

7. Homma Y, Ohtsuka Y, Tanimura K, et al. Can interstitial pneumonia as the sole presentation of collagen vascular diseases be differentiated from idiopathic interstitial pneumonia? Respiration. 1995;62(5):248-251.

8. Fischer A, Swigris JJ, du Bois RM, et al. Anti-synthetase syndrome in ANA and anti-Jo-1 negative patients presenting with idiopathic interstitial pneumonia. Respir Med. 2009; 103(11):1719-1724.

9. Fischer A, Meehan RT, Feghali-Bostwick CA, West SG, Brown KK. Unique characteristics of systemic sclerosis sine scleroderma-associated interstitial lung disease. Chest. 2006;130(4):976-981.

10. Travis WDCT, Koss MN, Rosado-de-Christenson ML, Muller NL, King TE Jr. Non-Neoplastic Disorders of the Lower
Respiratory Tract. Washington, DC: American Registry of Pathology and the Armed Forces Institute of Pathology; 2002.

11. Leslie KO, Trahan S, Gruden J. Pulmonary pathology of the rheumatic diseases. Semin Respir Crit Care Med. 2007; 28(4):369-378.

12. Song JW, Do KH, Kim MY, Jang SJ, Colby TV, Kim DS. Pathologic and radiologic differences between idiopathic and collagen vascular disease-related usual interstitial pneumonia. Chest. 2009;136(1):23-30.

13. Fujita J, Ohtsuki Y, Yoshinouchi T, et al. Idiopathic nonspecific interstitial pneumonia: as an "autoimmune interstitial pneumonia”. Respir Med. 2005;99(2):234-240.

14. Fukuoka JLK. Practical Pulmonary Pathology. A Diagnostic Approach. Philadelphia, PA: Churchill-Livingstone; 2005.

15. Kinder BW, Collard HR, Koth L, et al. Idiopathic nonspecific interstitial pneumonia: lung manifestation of undifferentiated connective tissue disease? Am J Respir Crit Care Med. 2007;176(7):691-697.

16. Mosca M, Neri R, Bombardieri S. Undifferentiated connective tissue diseases (UCTD): a review of the literature and a proposal for preliminary classification criteria. Clin Exp Rheumatol. 1999;17(5):615-620.

17. Vaz CC, Couto M, Medeiros D, et al. Undifferentiated connective tissue disease: a seven-center cross-sectional study of 184 patients. Clin Rheumatol. 2009;28(8):915-921. 